\title{
Medical Modeling and Fabrication of Surgical Guides
}

\author{
Ayna Zahoor ${ }^{1 *}$, S.S Banwait ${ }^{1}$, Nazir Ah Sheikh ${ }^{2}$ \\ ${ }^{1}$ Department of Mechanical Engineering, NITTTR Chandigarh, 160019, India \\ ${ }^{2}$ Department of Mechanical Engineering, NIT, Srinagar, 190006, India
}

\section{Corresponding Author:}

Corresponding Author: Ayna Zahoor

Mailing Address: ${ }^{1}$ Department of Mechanical Engineering, National Institute of Technical

Teachers' Training \& Research, Chandigarh, 160019, India

Email: aynazahoor35@gmail.com 


\begin{abstract}
Medical Modelling and Rapid Prototyping are being used extensively to produce accurate implants. Rapid Prototyping has been used in making 3D medical models. The models help in personalizing pre-surgical treatment and pre-operative planning.

Medical modelling is used for creation of high precision physical models from medical scans. The process includes collecting scans. The process includes collecting human anatomy data and optimizing the data for manufacturing and creating models using rapid prototyping. Computer aided software is used for prototyping. To produce high precision models, the modelling has to be in 3D. Therefore, a proper scanning method has to be used for each type of surgery. In this work we have taken a case study of fingers of hand to design and develop the 3D model for each case. We have designed, developed and fabricated one of the surgical guides for the fingers of hand. The design and fabrication was done in the 3D slicer and GrabCAD software independently. This work provides the approach and systematic procedure for the design and fabrication of surgical guide.
\end{abstract}

Keywords: Rapid Prototyping, Computer Aided Design, Reverse Engineering, Computed Tomography, Magnetic Resonance Imaging, Fused Deposition Modelling, Three Dimensional printing 


\section{Introduction}

From a medical point of view, the human body is both a subject and an object. The study of human form is the basis of all medicines that correct our dysfunction and degeneration. Based on these lofty goals, we have been working hard to apply the latest technology to medicine. Improve our treatment of all types of diseases. When unpleasant events such as illness or injury affect our physical health, they not only weaken our body, but also affect our mental health and to reproduce human anatomy with the infinite nuances we hope is a difficult and arduous task. Medical modelling and rapid prototyping are being used extensively to produces accurate implants, helps better surgical planning and patient counselling. Medical modelling can be done by using numerous available software's like 3D doctor, MIMICS, Slicer-3D, etc. However, for our project we are using open-source software, Slicer-3D. In recent years, rapid prototyping (RP) has been used to build highly accurate anatomical models from medical scan data. These models have proved to be a valuable aid in the planning of complex reconstructive surgery, particularly in maxillofacial and craniofacial cases. Typically, RP is used to create accurate models of internal skeletal structures on which operations can be accurately planned and rehearsed. In complex cases each implant is positioned successfully and fixed without complication. However, despite the successful clinical outcomes, the design process is highly involved, requires numerous interactions between the clinical and engineering teams, and it required multiple concept iterations; therefore, it could be argued to be cost-inefficient. With the intensification of competition and the adaptation of technology in the medical field, procedures for complex surgery have become simpler and more promising. The age of information technology does not necessarily make this process easier. The structure and rehabilitation of the human body can include any combination of dressing, rehabilitation, prosthetics, and surgery. The skills used range from techniques for restoring teeth to making artificial implants. Rehabilitation depends almost entirely on the skills and art of a small but highly dedicated community of health professionals. Nowadays, with improvement of survival rate and the increasing complexity of surgical procedures these people are facing increasing pressure. Therefore it is not surprising that medical science is seeking technology to save the workload and development, time and cost savings. Nowadays, surgeons are rapidly adopting various rapid prototyping (RP) solutions to guide the selection and actions of surgical procedures quickly and individually produce implants, prostheses, and orthopedic devices. At present, computer-aided design (CAD), reverse engineering (RE) and rapid prototyping (RP) play a leading role in the development of many rehabilitation aids and products suitable for the patient's anatomy. The thought of RE has perpetually been developed from the initial section or engineering called forward engineering (FE). The most distinction between the two engineering is that each result in an end product however both don't begin with a similar input. RE needs a product whereas FE wants an art. each begin with an idea of a new product preparation however metallic element starts from a style data that should be re modelled to a region whereas an available half is an input to the RE that has to be redesigned, re modelled and redeveloped. It typically plays an important role within the preparation of a brand-new model or a system with new functionality, technology and dimensions.

Rapid prototyping (RP) has been used in making 3D medical models. These models have proved to be valuable aid in personalize in pre-surgical treatment and pre-operative planning. RP is used to create accurate models of internal skeletal structures which aid in planning and rehearsing the medical procedures. By using these models complex surgeries are performed with implants positioned and fixed without complications. Surgeons are rapidly adopting 1 various RP solutions to guide and actions of surgical procedures

\subsection{Applications}

Rapid Prototyping and Medical Modelling has been used in the design and development of medical devices and instrumentation. These include the hearing aids and surgical aid tools where utilization is better.

There have been improvements to the fields of prosthetics and implantation as we are using rapid prototyping in making prostheses and implants like hip sockets, knee joints and spinal implants for quite some time.

The improvements in the material availability and desirable changes in the properties of materials had a significant influence on the quality of prostheses and implants. Nowadays it is easy to manufacture custom implants by rapid prototyping techniques. The Modelling and 3D printing has improved the way of learning the surgical procedures and planning the complex surgical operations. This is very important role of RP technologies in medicine which enable pre-surgery planning. The use of $3 \mathrm{D}$ medical models helps the doctors to plan and perform complex surgeries. The surgical guides' makes surgery more efficient by giving opportunity to study the bony structures of the patient before the surgery, which increases surgical precision, reduce time of procedures and risk during surgery.

Using segmentation technique we can mark different structures in different colors in a 3D physical model which can be very useful for surgery planning and better understanding the procedure. It will be also useful for the teaching and learning purposes e.g. in cancer surgery where tumor tissue can be clearly distinguished 
The models can be used for the teaching medical students as well as for the researchers, as these models can be made in many colors and provide a better illustration of anatomy and allow viewing of internal structures. They are also used as teaching simulators.

RP technologies gave significant contribution in Design and manufacturing biocompatible and bioactive implants and tissue engineering. These include the direct manufacture of bioactive implants. RP systems like fused deposition modelling (FDM), 3D printing (3-DP) and selective laser sintering (SLS) have been proved to be convenient for making porous structures used in tissue engineering. In this three-dimensional scaffolds of various geometric shapes are fabricated which are used to repair defects caused by accidents, surgery etc.. FDM, SLS and 3DP can be used to fabricate a functional scaffold directly but RP systems can also be used for manufacturing a sacrificial mold to fabricate tissue-engineering scaffolds.

The RP technology selection has to be in accordance with the purpose of model itself as well as demanding accuracy, surface finish, visual appearance of internal structures, number of desired colors in the model, strength, material, mechanical properties, etc. The 3D virtual model in STL format is fed to the RP software for production of 3D physical model. The quality of physical model is not only dependent on the quality of STL file but also on orientation of the model in RP machine. Also it is dependent on parameters for building the model in the machine.The fabricated medical model is validated by surgeons and if there are no errors the model is ready for application.

\subsection{Materials Used}

There are a number of materials which can be used for medical applications of RP. The material selection depends on the purpose of model, e.g. planning procedures, implants, surgical tools, prostheses, tissue scaffold etc. It also depends on the process or RP technique to be used as per the material selection. The materials should be also show biological compatibility.

\subsection{Lack of Regulation}

The biomedical field is one of the areas in which 3D printing has already shown its potentialities and we hope in near future this will be one of the important elements in solving the human health problems. Nowadays, despite the fact that additive manufacturing offers a great potential for the manufacturing but the 3D-printing products do not have a proper legal status that defines them, both for implantable and non implantable devices.

\subsection{Rapid Prototyping}

A complete RP process includes the creation and slicing of a virtual computer model usually followed by the layer-by-layer fabrication process. This is also known as solid freeform fabrication manufacturing, automated manufacturing, multi-layer manufacturing, etc. It consists of a set of technologies that can use computer-aided design (CAD) models and convert them into physical shapes or parts. The process is automatic, usually independent of the geometry of the model, and does not require any special tools or accessories. Complex 3D (3-D) contours are quantified as a stack of finite thickness layers or 2D sections. When these layers are very thin, the finished part will be accurate enough to suit various applications. The details of the RP can be characterized by a step effect, which approximates its original shape and is most obvious on a gentle slope proposed a more complete classification of the RP process, focusing on the initial state of the raw materials used, namely the production of solids, liquids and powders. Rapid Prototyping (RP) and rapid manufacturing (RM) are two common terms used to describe additive manufacturing technology before the term "additive manufacturing" is used. Compared with RP and RM phrases, AM is considered to be a more general term that directly reflects the processing strategy of this advanced manufacturing technology. The initially developed additive manufacturing strategies include stereo lithography, roll-to-roll, enamel deposition modelling, 3D printing, and selective laser sintering. Few existing technologies can produce metal parts, and these metal parts are currently being proven and used in the industry. There are three main medical RP applications, including: design and manufacturing of bio models, surgical aid tools and implants, development of surgical training models and medical devices and design and manufacturing of scaffolds for tissue engineering. Additive production strategies have been first added with inside the scientific area, extra especially with inside the subject of dentistry, with inside the 1990s. The first biomedical fashions have been to start with constructed with a didactic goal and, later, with a surgical purpose. In current years, RP approach studies advanced unexpectedly in the molding material and the forming process. This technology is now not used completely for prototyping, however may be used to manufacture actual useful parts. Therefore, RP is becoming greater appealing in dental applications. RP strategies can additionally be used to design, develop, and manufacture dental prostheses including copings, crowns, and glued partial dentures (FPDs). Traditionally, dental prosthesis fabrication involves much laborintensive and time-eating hands-on work through dentists and technicians. Dental prosthesis fabrication has additionally been greatly dependent on the abilities of dentists and technicians. Compared to conventional methods, new dental prosthesis work may be fabricated through RP strategies layer through the layer at 
have developed with inside the market place with numerous competencies and advance each in phrases of fabric in addition to speedy transport and effectiveness. The not unusual place maximum methods consist of sintering, Powder-based technology, Fused Deposition modelling, laminated object manufacturing and so on. The primary intention of the RP machines is the rapid manufacturing of custom designed merchandise in case of scientific printing. The evolution of the methods has been thru the 80s when SLA and Selective laser sintering have been initiated with the aid of using 3D structures and DTM Co of Austin. Classification of prototyping is shown below in figure

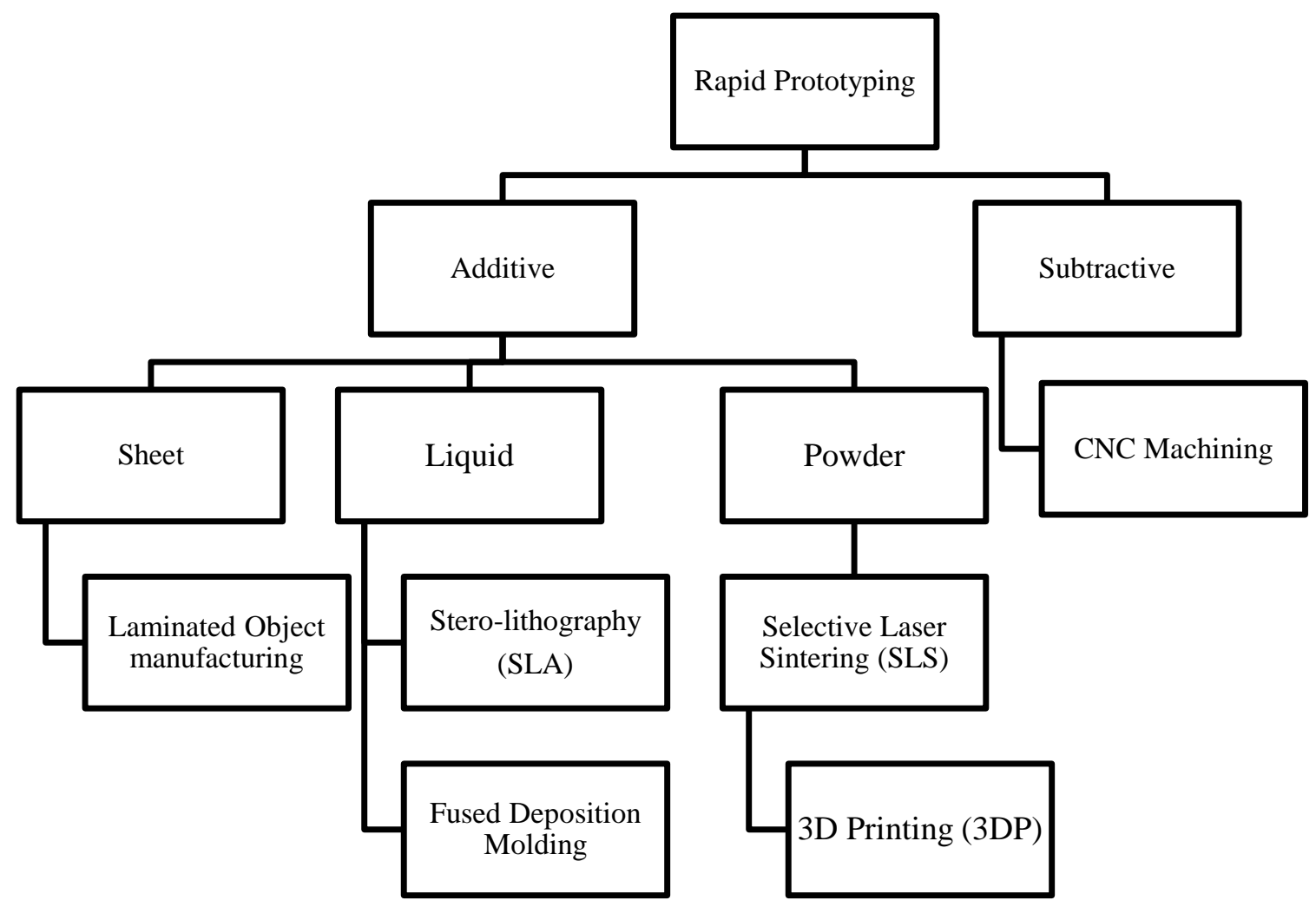

Fig. 1.2 Classification of Rapid Prototyping 


\section{Surgical Guides}

Our research suggests that surgical guides are well incorporated in orthopedic surgery, spinal surgery, maxillofacial surgery, and dental surgery with more than half of the selected studies of our review mentioning the use of guides. Increased procedural complexity and less-experienced surgeons will prefer to use the surgical guides. Apart from clinical results, patient-specific guides also reduce the number OR time. The more surgeons are used to the surgical guided procedures the more reduction in OR time. Although there are not much support to the claims that the surgical guided procedures are cost-effective but in some of the surgeries like in case of knee cost effectiveness is prominent. Based on our survey the surgical guides reduce operation room time and improve medical outcomes for spinal and cranial surgeries. This is because of the medical modelling and the translation of the preliminary surgery by surgical guides. As the literature survey carried out by different researchers the spinal surgical guides translate the surgical planning precise and accurately and make the outcomes less dependent on the surgeon's experience. Similar results are seen with the use of guides during dental surgeries. Some author's question that the surgical guides should be used only in case of complex cases to reduce the associated costs. A number of case studies reflect the maturity of the technological application of medical modelling and surgical guides. Some of these studies and their recommendations is summarized as under:

Bibb et al reported that software system (CAD) and tailored production of surgical guides are a certain means that of transferring computer-aided about to surgery. That typically need metal reinforcement to stop harm from bits. This text aims to gift case studies examining the applying of selective optical device fusion (SLM) within the direct fabrication of stainless-steel surgical guides. Verify whether or not the potential edges of inflated stiffness, increased wear resistance (reset), and easier autoclaving will be realized in the field [25].

Chen et al presented a low-priced methodology of making made-to-order 3D-printed bone entrench that are tailored to the dissection of subjective animals. We have affinity to performed pre-surgical computed tomography (CT) and magnetic resonance (MR) scans to come up with three-dimensional (3D) models of the cranium and brain. We have an American 3D modelling code to style implantable skull posts, guild, and a pedestal anchorage base, moreover as surgical course guides to help us throughout surgery. Prototypes were made up of plastic or resin, whereas implants or ingrains were 3D-printed in titanium. The implants live through post-processing and meet with a coating of osteocompatible material to push bone integration [26].

Ganz and Scott This article illustrates the benefits of using CT-based templates through various clinical presentations. Methods for single and multiple tooth applications in mandibular and maxillary arches were presented. As implant medical specialty is evolving toward accelerated treatment protocols, with instant or delayed practical and non-functional loading, the consequence of presurgical coming up with paramount. The paradigm for restorative-driven graft placement works best once usher are wont to transfer data from the specified conceive to the surgical reality. The appearance of computed tomography (CT) imaging, and CT-derived surgical templates afford clinically needed enhancements in exactness, time efficiency, and trimming in surgical error, benefiting the patient, surgeon, restorative dentist. Continuing advances within the progressive code applications that modify increased planning give clinicians the vision necessary to confinement the impulse results, whereas serving as an magnificent communication tool between all members of implant team [27].

Pieralli et al investigate to see the accuracy of a surgical progress for oral implant placement victimization guides factory-made by means that of amalgamate deposition modelling (FDM) from a perishable and castrate biopolymer filament. Furthermore, the good thing about metal sleeve inlay ought to be assessed. A surgical guide was map out for the installation of two implants within the domain of the second premolar (SP) and second molar (SM) in an exceedingly jaw typodont model. There was no effect of metal sleeves on axial, vertical, and mesial accuracy. Concerning accuracy, biopolymer-based surgical guides made by FDM gives similar accuracy than SLA. Antileukemic tests are necessary to uphold their biocompatibility within the oral environment [28].

Ganry et al created the 3D modelling of the reformation mandible and its surgical ushers. Four succeeding speciate open-source computer code were accustomed for 3D modelling: OsiriX®, Meshlab®, Netfabb ${ }^{\circledR}$ and Blender ${ }^{\circledR}$. Digital Imaging and Communications in medication (DICOM) information on patient bone and fibula, acquired with a processed imaging (CT) scan. This new strategy can improve surgical guidance in oral and craniomaxillofacial surgery [29].

Giordano et al Used the laser scanning to evaluate, by indirect methods, the delicacy of computer-designed surgical guides in the oral implant assisted rehabilitation of partially or completely edentulous patients. Five implant-assisted restorations were performed for a total of 23 implants using computer-aided surgical guides 
fabricated with the help of casting and developed by muco-compressive and muco static impressions. For all cases, virtual operation planning from 3D models. The implants were inserted into the surgical operative models at the location defined in the virtual planning, these positions were recorded by three-dimensional optical laser scanning and weigh up with the laser scans of the intraoral suspicion [30].

Sarment et al reported to rival the exactness of a conventional surgical guide thereto of a stereolithographic surgical guide. CT scanning of epoxy toothless lower jaw was enacted employing a cone beam CT scanner with inflated similar spatial resolution, whereas make-up for five implants on all sides of the jaw was performed using a commercially accessible package.Surgeons accomplished osteotomies on a jaw alike to the inceptive model. On the proper side a typical surgical guide (control side) was used, and on the left side a stereolithographic guide was used (test side). Every jaw was then CT scanned, and a registration technique was appertained to match it to the first forethought. Measurements encompassed distances between organized implants and actual osteotomies [31]

Gill et al has taken up a small age girl patient for cranioplasty having cranial defect. Authors used the rapid prototyping technology for development of exact individual templates for patient and surgery was performed successfully by using Simplex implants. 3D slicer software was used for DICOM data. Generation of 3D model was performed in two step first one is generating of standard language file of head with defect. Second one is implant model which was generated by reverse engineering, mirroring, Boolean expression, cleaning and slicing, printing using RP and then design of custom implant [32].

Poukens et al suggested that navigation and the use of surgical guides are both unerring enough for surgical resolutions. Two infusions exist to convey the computer plan to the operating theatre: navigation systems and surgical guides. The use of navigation systems is a fancier field itself [33]

Ballard et al generated an economic analysis of the cost-saving utter of 3D printed intellectual models and surgical guides in orthopedic and maxillofacial surgical exercise. A targeted literature search known operating cost-per-minute and reports that quantified time.3D printed anatomic models or surgical guides were reviewed and catalogued. Based on the literature-based money analyses, medical 3D printing seems to scale back hospital room prices and shortening procedure times. Whereas resource-intensive, 3D printed constructs utilized in patients' operative care provides sizable downstream worth to health systems [34].

Ochi et al evaluate the correctness of implant placement with mucosa-supported surgical guides in edentulous mandibles and to find the factors affecting accuracy. The implant placement was replicated on the preoperative CT images, and CAD/CAM technology was used to make mucosal-assisted surgery templates for six edentulous models and fifteen edentulous patients. By comparing the planned image and the postoperative image, the deviation between the actual position of the implant and the outlined position is calculated. Based on the upshots, it can be gathered that the mucosal assisted surgery guidelines are very accurate, and bone density and mucosal thickness will affect the correctness [35].

Krishnan et al reports the improvements in the surgical technique of knee prostheses. Recently, there has been interest in using magnetic resonance imaging (MRI) and CT for three-dimensional (3D) preoperative planning. 3D imaging is increasingly used to create customized patient models, surgical guides and implants for keen prostheses. Users of Patient-Oriented Instruments claim that they have achieved the best balance between technology and conventional surgery by reducing the complexity of traditional alignment and sizing instruments. Therefore, the advantages of accuracy and precision required by the computer navigation method are achieved without the disadvantages related to additional intraoperative equipment, new skills, or operation time [36].

\section{Methodology:}

The first step in design and fabrication of the surgical guide is image acquisition and the target area selection. There are different scanning mechanisms used in acquiring the image of the patient's region of interest but for all the scanning mechanisms we have to obtain the scan in DICOM format. The next step is segmentation i.e. transforming the DICOM to STL file. For the identification of the specialist is to identify the region of interest, volume rendering is done using the slicer 3D modeling software. The region of interest is identified by Thresholding, segmentation and labelling. A CAD modeling tools are used to design 3D model of the part conforming to the anatomical geometry. The 3D CAD model file is converted to STL format. The STL file is then send to the $3 \mathrm{D}$ printer for fabrication purposes. A complete workflow of the methodology is used: 
8

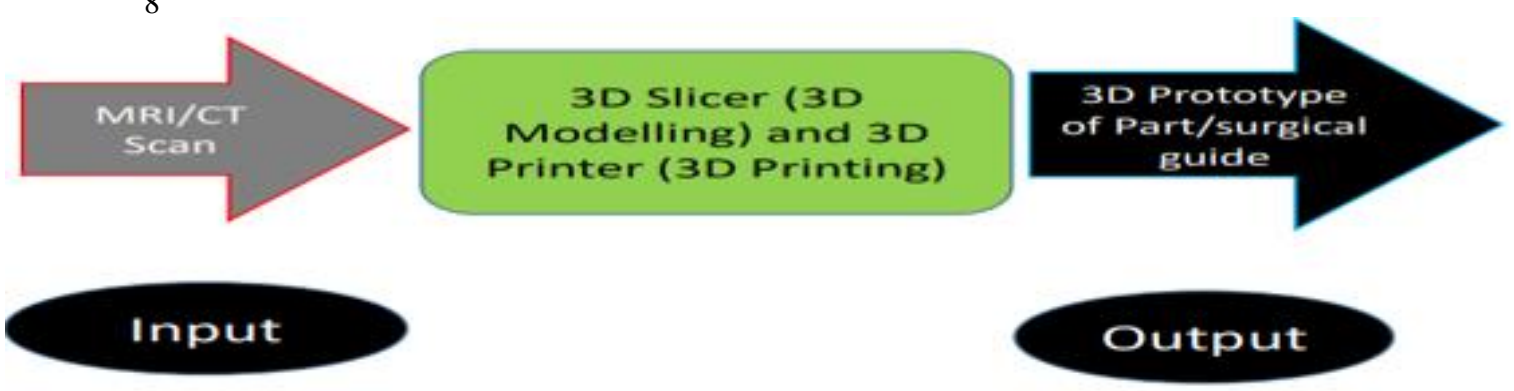

Figure 3.2:3D Printing and 3D modelling functional diagram

The whole process of design and fabrication is based on the selection of region of interest by the specialist doctor who identifies which part of the body is scanned, its size and other features. Once the image of the patient's part is loaded the other operations are all dependent on the software used for the analysis purposes. In our work we have used 3D slicer. In the 3D slicer the image is loaded as shown in fig 3.3

\section{Case Study of Hand}

The Figures (4.1 to 4.3) shows the medical modelling of hand. The resultant images also depict the change in the medical model view by changing the threshold values. The models have been fine tuned to clarify the region of interest, so that the visualization of the target area is increased.

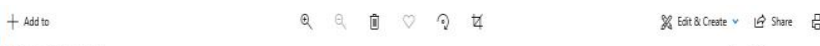

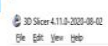

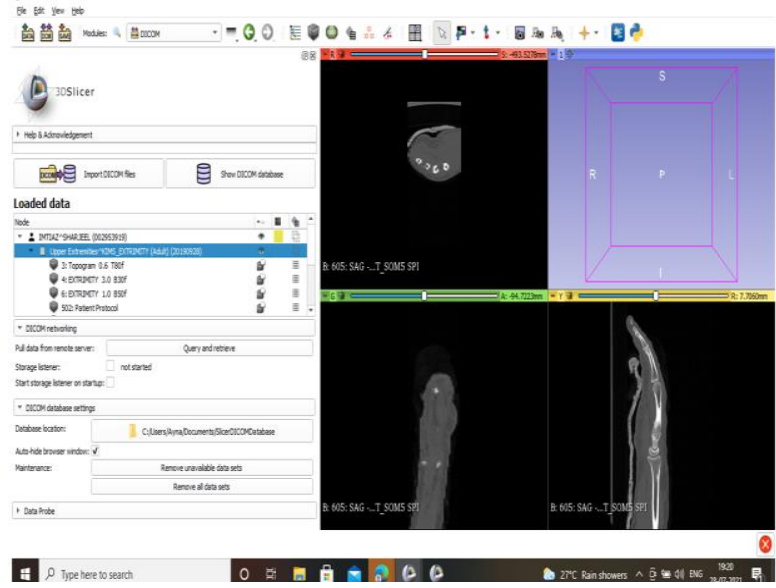

Figure 4.1: CT scan of the hand of patient

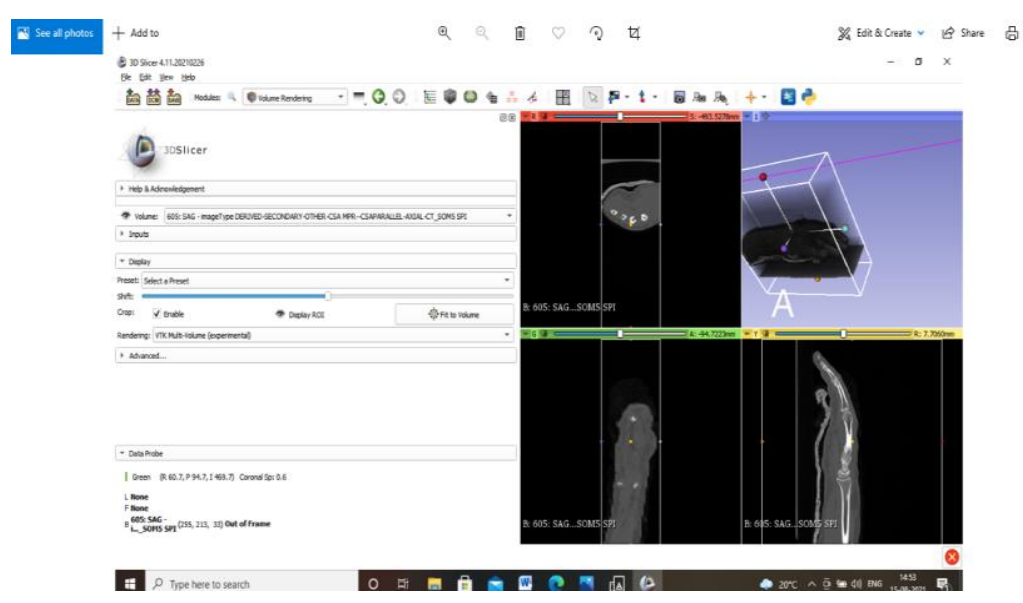

Figure 4.2:3D Model after Volume Rendering 


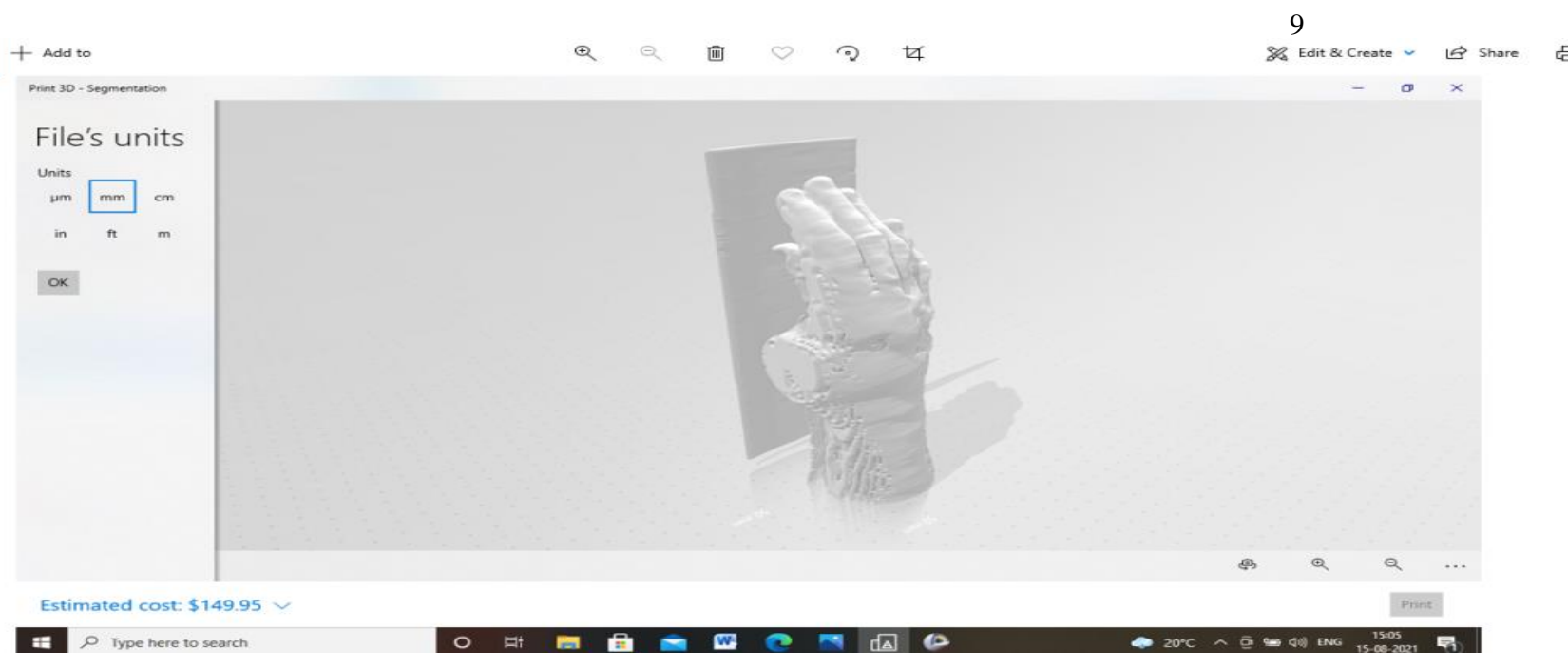

Figure 4.3: STL file of hand

\subsection{Surgical Guide for the Fingers of Hand:}

As shown in Figure 4.5 the image of medical modelling views of hand of the patient. The image characteristics and threshold values are changed as per the desired target area. The generated view is shown in Figure 4.5. The Thresholding is done to desired levels for clarity and the target areas are identified as shown in figure 4.6. The Figure 4.6 gives a better visualization of the medical model as compared to the Figure 4.5 due to thresholding. The region of interest is identified as shown in top right corner of the Figure 4.7 and 4.8. For making the precise surgical guide or implants we narrow done on the region of the interest for better identification of the parts used for further processing using the segmentation editor tool a label mapping. 3D model of the hand is generated as shown in Figure 4.9 the 3D solid model is designed for the surgical procedure as shown in Figure 4.10. For the defective part to be cut or removed from the main part holes are to be drilled for fixation of any implant on the bone at the specified location at any specified angle. Hence the surgical guide is fabricated for the hand using segmentation editor tool of the 3D slicer. The developed model is shown in Figure 4.11

\subsection{Generation of 3D printable file of the designed 3D model:}

After developing the $3 \mathrm{~d}$ model of the hand the next important step to be followed is to generate a $3 \mathrm{D}$ printable file in STL or obj. format generally. The fingers of hand were chopped off because the size of hand was big and the fortus $450 \mathrm{mc}$ available in the lab could not print the complete part as the build volume of the machine is small. The screenshot of the input to the fortus $450 \mathrm{mc}$ printer for the hand is shown (F12)

\subsection{Generation of 3D printable file of the designed 3D model of the surgical guide:}

After developing the 3D model of the surgical guide the next important step to be followed is to generate a 3D printable file in STL or obj. format generally. The screenshot of the input to the fortus $450 \mathrm{mc}$ for the surgical guide is shown in fig 5.33 


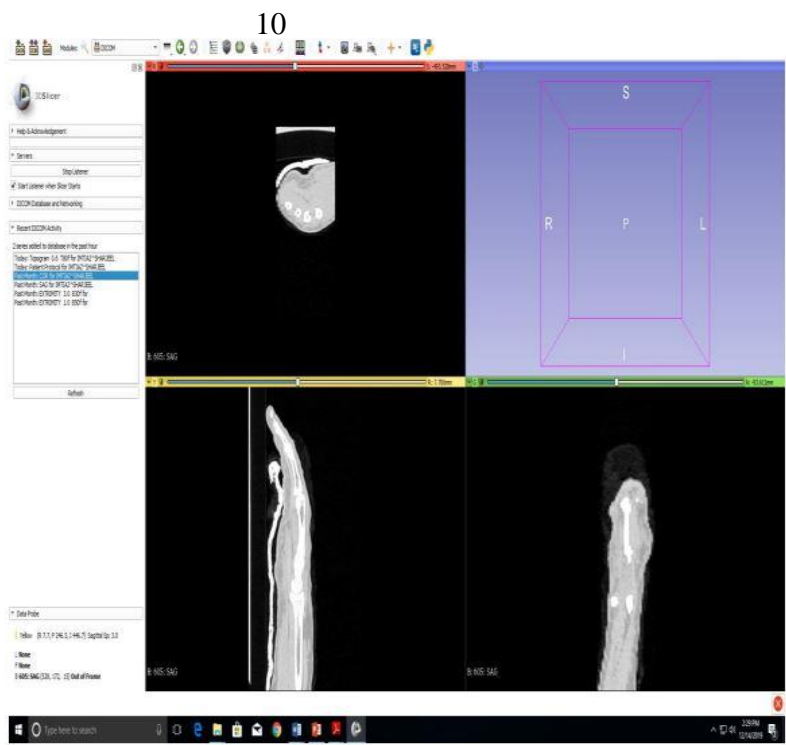

Fig 4.4: Image of medical modelling views

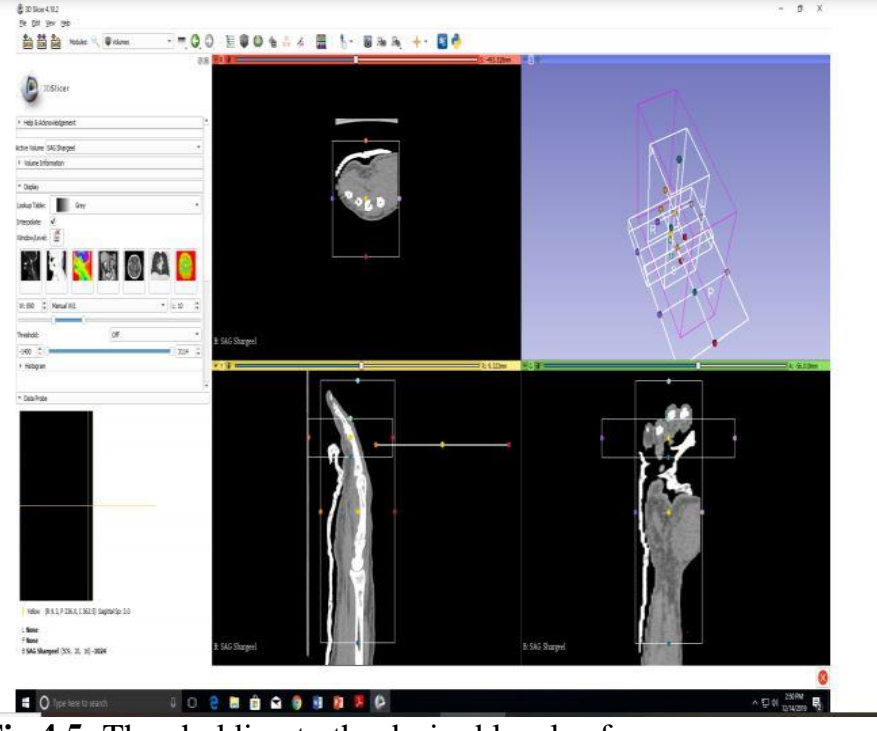

Fig 4.5: Thresholding to the desired levels of Clarity to identify the parts of interest of bones and muscles
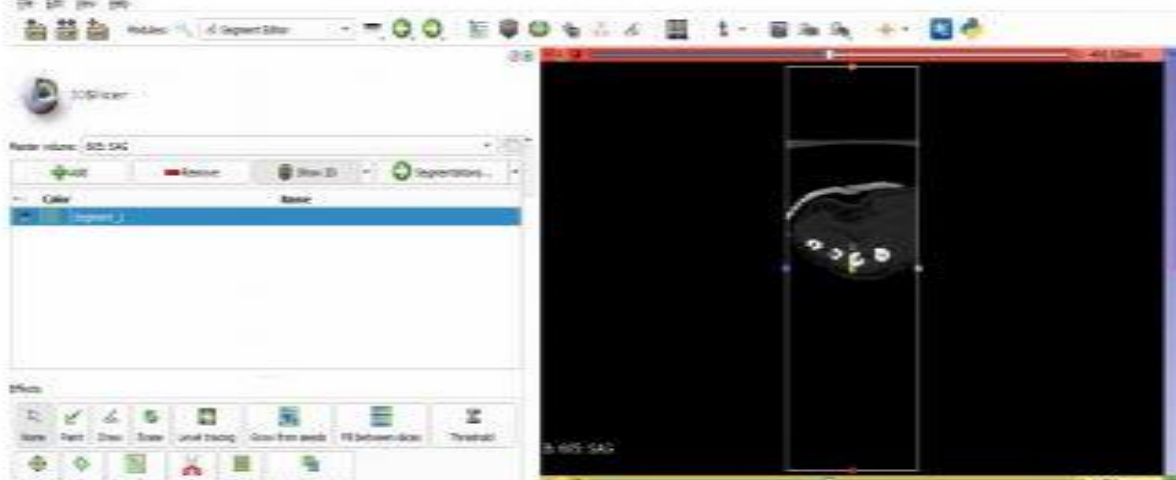

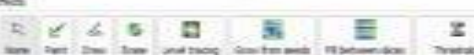

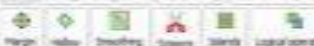

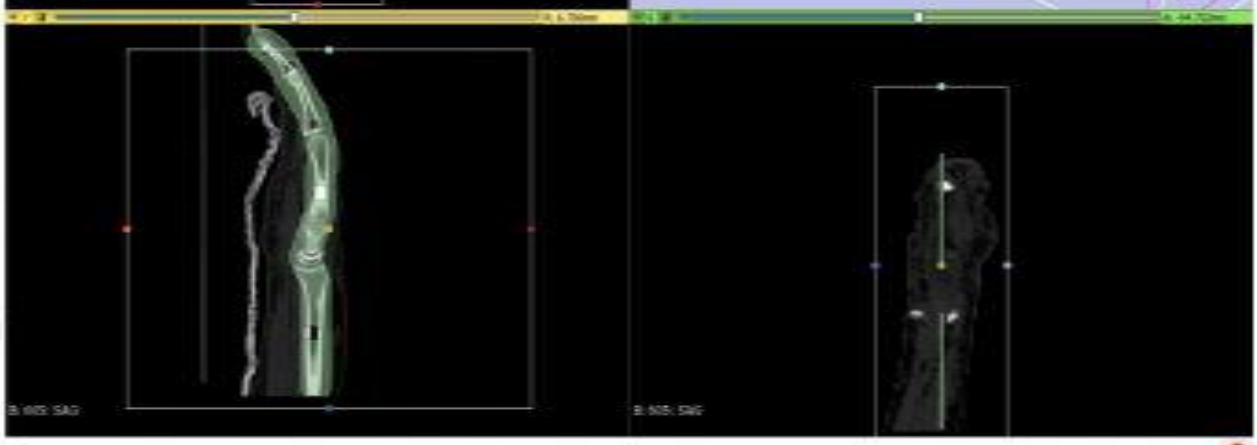

Fig 4.6: Model of the Surgical Guide shown in top right corner 


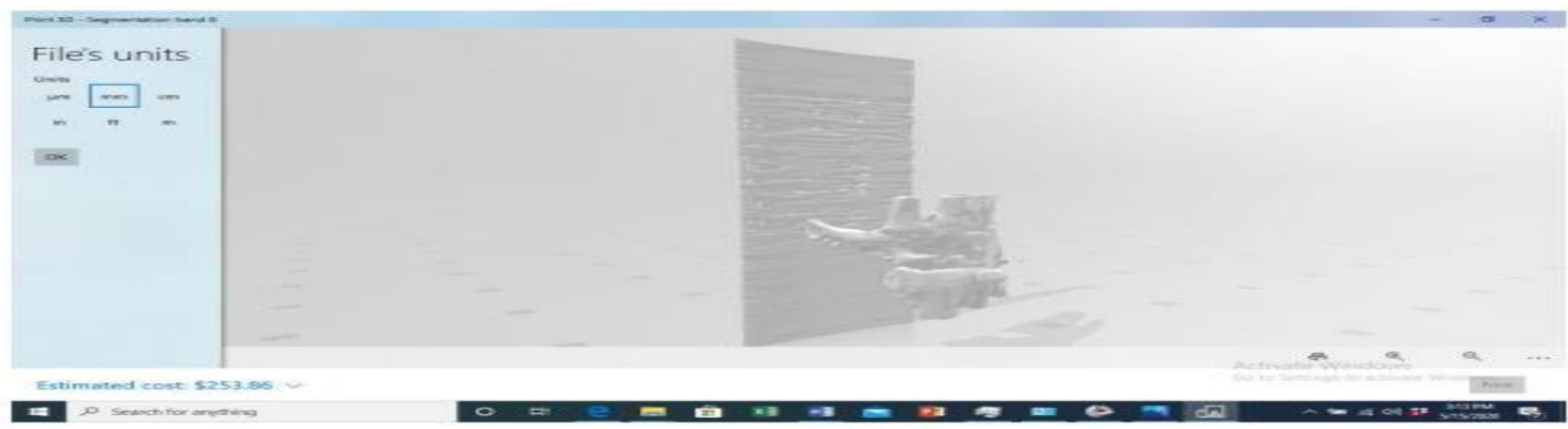

Figure 4.7: Input of the chopped fingers of the hand in STL file format used for printer.

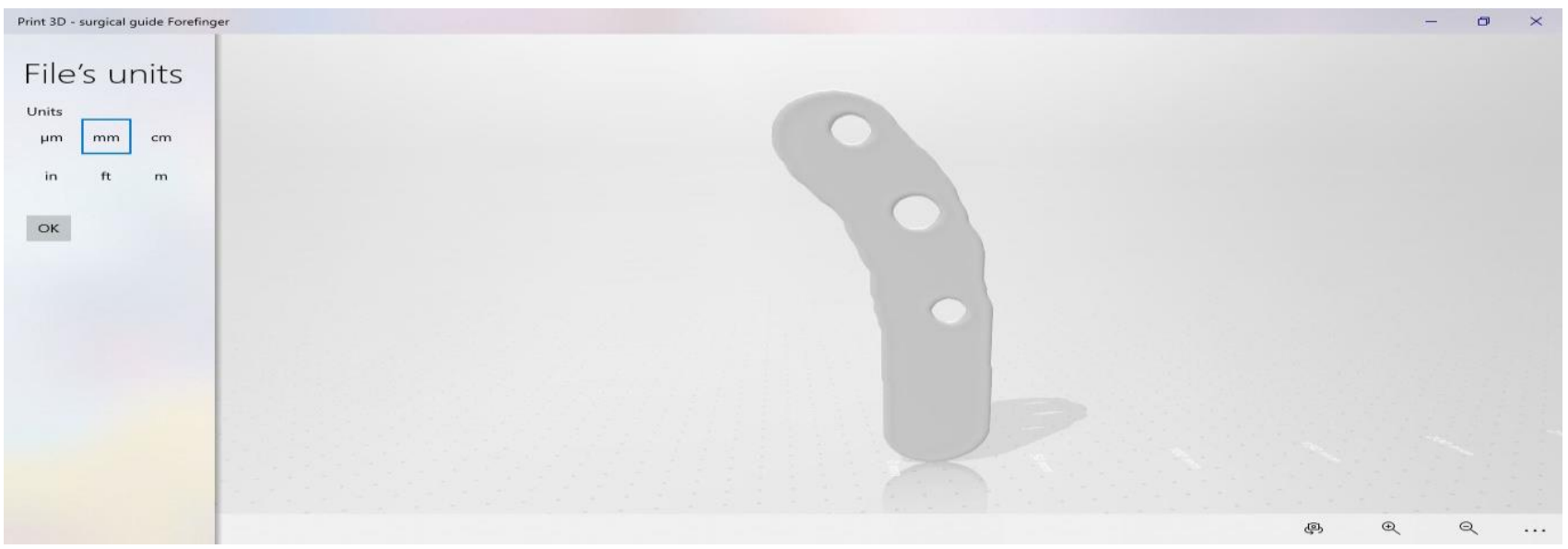

Figure 4.8: Input of the surgical guide in the STL file format to the printer

Figure 4.9 shows the final prototype of the model

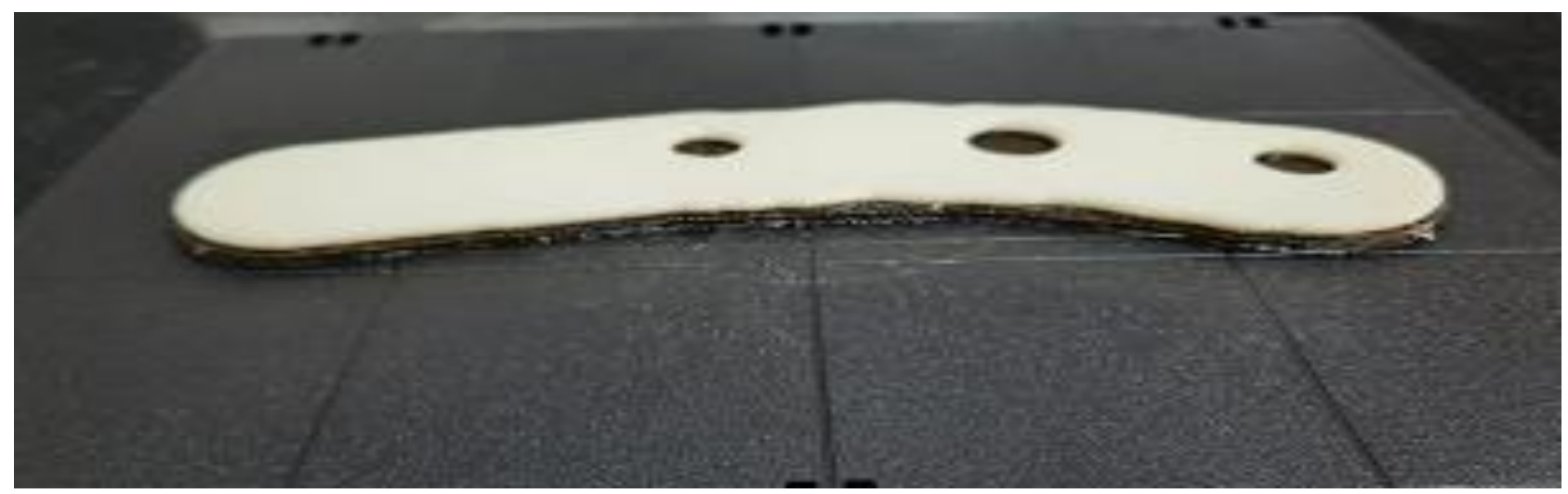

Fig 4.9: Final Prototype 
12

\section{Conclusion}

The design and fabrication of surgical guides is dependent on the selection of scanning process for image acquisition. The region of interest has to be decided in consultation with the surgeon. The process of identifying the region of interest is by thresholding, segmentation and labelling. CAD modelling tool is used for the 3D modelling. The 3D CAD model file is converted to STL file format.

The process of medical modelling is influenced by the material selected. According to the material selected the process is also identified and followed in the software and at the fabrication level.

The process of design and fabrication is dependent on the region of interest i.e. the part of body selected for surgery or implants. The material of the surgical guide depends on the tissue involved. Support material is accordingly selected to avoid any distortion in the printed model. The $3 \mathrm{D}$ printer is to be selected as per the material and the process.

The surgical guides in general help to improve the clinical performance. These have the potential to improve the research knowledge and the skills of the surgeons, improve the understanding between the patient and the surgeon, and improve the design of the implantable devices and surgical tools. The medical models also facilitate the visualization and learning and to educate the medical students and practising doctors.

Finally in our case under study, design and fabrication process of surgical guide of hand fingers, the surgical guide help to reduce the errors on part of the surgeons during the surgical operation. This leads to less surgical time and high success rate.

\section{References}

1. Regulation (EU) 2017/745 of the European Parliament and of the Council of 5 April 2017 on medical devices, amending Directive 2001/83/EC, Regulation (EC) No 178/2002 and regulation (EC) No 1223/2009 and repealing Council Directive 90/985/EEC and 93/42/EEC.

2. Article 2, Comma 3 of the Regulation (EU) 2017/745.

3. Article 20, Comma 1 of the Regulation (EU) 2017/745.

4. Article 10 of the Regulation (EU) 2017/745.

5. Article 2, Comma 5 of the Regulation (EU) 2017/745

6. S. Dunham, Surgeon's Helper: 3D Printing is Revolutionizing Health Care (Op-Ed), SmarTech Markets, Charlottesville, VA, USA, 2015, https://www.livescience.com/49913-3dprintingrevolutionizing-health-care.html.

7. lgh Eshkalak S, Rezvani Ghomi E, Dai Y, Choudhury D, Ramakrishna S: The role of threedimensional printing in healthcare and medicine. Materials \& Design 2020 194:108940. doi:https://doi.org/10.1016/j.matdes.2020.108940. * This a comprehensive study that presents thoroughly different additive manufacturing methods and their applications in healthcare.

8. Tuan D.Ngo , Alireza Kashani, Kate T.Q, Nguyen, David Hui," Additive manufacturing (3D printing): A review of materials, methods, applications and challenges", Composite Part B, Journal, 2018

9. Shi B, Huang H: Computational technology for nasal cartilage-related clinical research and application. International Journal of Oral Science 2020 12:21. doi:10.1038/s41368-020-00089-y

10. Yu H, Zhao Y, Li J, Luo T, Gao J, Liu H, Liu W, Liu F, Zhao K, Liu F, Ma C, Setz JM, Liang S, Fan L, Gao S, Zhu Z, Shen J, Wang J, Zhu Z, Zhou X: Minimal invasive microscopic tooth preparation in esthetic restoration: a specialist consensus. International Journal of Oral Science 
11. Bester C, Razmovski T, Collins A, Mejia O, Foghsgaard S, Mitchell-Innes A, Shaul C, Campbell L, Eastwood H, O’Leary S: Four-point impedance as a biomarker for bleeding during cochlear implantation. Scientific Reports 2020 10:2777. doi:10.1038/s41598-019-56253-w

12. Tuomi J, Paloheimo K-S, Vehviläinen J, Björkstrand R, Salmi M, Huotilainen E, Kontio R, Rouse S, Gibson I, Mäkitie AA: A Novel Classification and Online Platform for Planning and Documentation of Medical Applications of Additive Manufacturing. Surgical Innovation 2014 21:553-559. doi:10.1177/1553350614524838

13. Kilian D, Ahlfeld T, Akkineni AR, Bernhardt A, Gelinsky M, Lode A: 3D Bioprinting of osteochondral tissue substitutes - in vitro-chondrogenesis in multi-layered mineralized constructs. Scientific Reports 2020 10:8277. doi:10.1038/s41598-020-65050-9

14. Durfee WK, Iaizzo PA (2019) Chapter 21 - Medical Applications of 3D Printing. In: Iaizzo PA (ed) Engineering in Medicine. Academic Press, pp 527-543. doi:https://doi.org/10.1016/B978-012-813068- 1.00021-X

15. Hoang D, Perrault D, Stevanovic M, Ghiassi A: Surgical applications of three-dimensional printing: a review of the current literature \& how to get started. Annals of translational medicine 20164

16. Ligon SC, Liska R, Stampfl J, Gurr M, Mülhaupt R: Polymers for 3D Printing and Customized $\begin{array}{lllll}\text { Additive } & \text { Manufacturing. } & \text { Chemical } & \text { Reviews } & \end{array}$ doi:10.1021/acs.chemrev.7b00074

17. Lele A (2019) Additive Manufacturing (AM). In: Lele A (ed) Disruptive Technologies for the Militaries and Security. Springer Singapore, Singapore, pp 101-109. doi:10.1007/978-981-133384-2_5

18. Trenfield SJ, Awad A, Madla CM, Hatton GB, Firth J, Goyanes A, Gaisford S, Basit AW: Shaping the future: recent advances of 3D printing in drug delivery and healthcare. Expert Opinion on Drug Delivery 2019 16:1081-1094. doi:10.1080/17425247.2019.1660318. * This publication provide a state of the art in applications of drug delivery and medicine using 3D printing and 4D printing.

19. Muehlenfeld C, Roberts SAJD, Engineering DPiBAP, Manufacturing A: 3D/4D Printing in Additive Manufacturing: Process Engineering and Novel Excipients. 2019:1-23

20. Wu J-J, Huang L-M, Zhao Q, Xie TJCJoPS: 4D printing: history and recent progress. 2018 36:563575

21. Gao B, Yang Q, Zhao X, Jin G, Ma Y, Xu F: 4D Bioprinting for Biomedical Applications. Trends in Biotechnology 2016 34:746-756. doi:https://doi.org/10.1016/j.tibtech.2016.03.004

22. Lee J-Y, An J, Chua CKJAMT: Fundamentals and applications of 3D printing for novel materials. 2017 7:120-133

23. He Y, Yang F, Zhao H, Gao Q, Xia B, Fu J: Research on the printability of hydrogels in 3D bio printing. Scientific Reports 2016 6:29977. doi:10.1038/srep29977

24. Tan D, Nokhodchi A, Maniruzzaman MJD, Engineering DPiBAP, Manufacturing A: 3d and 4d printing technologies: innovative process engineering and smart additive manufacturing. 2019:2552.

25. Bibb, R., Eggbeer, D., Evans, P., Bocca, A., \& Sugar, A. (2009). Rapid manufacture of custom3, fitting surgical guides. Rapid Prototyping Journal. 
14

26. Chen, X., Possel, J. K., Wacongne, C., Van Ham, A. F., Klink, P. C., \&Roelfsema, P. R. (2017). $3 \mathrm{D}$ printing and modelling of customized implants and surgical guides for non-human primates. Journal of neuroscience methods, 286, 38-55.

27. Ganz, Scott D. "Presurgical planning with CT-derived fabrication of surgical guides." Journal of oral and maxillofacial surgery 63.9 (2005): 59-71.

28. Pieralli, S., Spies, B. C., Hromadnik, V., Nicic, R., Beuer, F., \&Wesemann, C. (2020). How Accurate Is Oral Implant Installation Using Surgical Guides Printed from a Degradable and SteamSterilized Biopolymer?. Journal of Clinical Medicine, 9(8), 2322.

29. Ganry, L., Hersant, B., Quilichini, J., Leyder, P., \&Meningaud, J. P. (2017). Use of the 3D surgical modelling technique with open-source software for mandibular fibula free flap reconstruction and its surgical guides. Journal of stomatology, oral and maxillofacial surgery, 118(3), 197-202.

30. Giordano, M., Ausiello, P., \&Martorelli, M. (2012). Accuracy evaluation of surgical guides in implant dentistry by non-contact reverse engineering techniques. Dental Materials, 28(9), e178e185.

31. Sarment, D. P., Sukovic, P., \&Clinthorne, N. (2003). Accuracy of implant placement with a stereolithographic surgical guide. International Journal of Oral \& Maxillofacial Implants, 18(4).

32. Branemark, P., \& De Oliveira, M. F. (Eds.). (1997). Craniofacial prostheses, anaplastology and osseointegration (pp. 101-110). Carol Stream, IL: Quintessence Publishing Co. Inc

33. Poukens, J., Verdonck, H., \& de Cubber, J. (2005). Stereolithographic surgical guides versus navigation assisted placement of extra-oral implants (oral presentation), 2nd international conference on advanced digital technology in head and neck reconstruction, Banff, Canada (p. 61).

34. Ballard, D. H., Mills, P., DuszakJr, R., Weisman, J. A., Rybicki, F. J., \& Woodard, P. K. (2020). Medical 3D printing cost-Savings in Orthopedic and Maxillofacial Surgery: cost analysis of operating room time saved with 3D printed anatomic models and surgical guides. Academic radiology, 27(8), 1103-1113.

35. Ochi, M., Kanazawa, M., Sato, D., Kasugai, S., Hirano, S., \&Minakuchi, S. (2013). Factors affecting accuracy of implant placement with mucosa-supported stereolithographic surgical guides in edentulous mandibles. Computers in Biology and Medicine, 43(11), 1653-1660.

36. Krishnan, S. P., Dawood, A., Richards, R., Henckel, J., \& Hart, A. J. (2012). A review of rapid prototyped surgical guides for patient-specific total knee replacement. The Journal of bone and joint surgery. British volume, 94(11), 1457-1461. 